\title{
Association between uncontrolled eating and caudate responses to reward cues
}

\author{
Patrícia Bado ${ }^{1-2 \bowtie}$, Jorge Moll $^{1}$, Bruno P. Nazar ${ }^{3}$, Ricardo de Oliveira-Souza ${ }^{1,4}$, Raquel da Costa ${ }^{1}$, Gail Tripp ${ }^{5}$, Paulo \\ Mattos $^{1,3}$, and Emi Furukawa ${ }^{5}$ \\ ${ }^{1}$ D'Or Institute for Research and Education (IDOR), Rio de Janeiro, Brazil \\ ${ }^{2}$ Morphological Sciences Program, Federal University of Rio de Janeiro, Rio de Janeiro, Brazil \\ ${ }^{3}$ Federal University of Rio de Janeiro, Rio de Janeiro, Brazil \\ ${ }^{4}$ Departments of Neurology and Psychiatry, Federal University of the State of Rio de Janeiro, Rio de Janeiro, Brazil \\ ${ }^{5}$ Okinawa Institute of Science and Technology Graduate University (OIST), Okinawa, Japan
}

\begin{abstract}
Reward sensitivity has been hypothesized to play a significant role in a range of eating behaviors, including overeating. Previous functional magnetic resonance imaging (fMRI) findings in overweight individuals indicate heightened responses to food, but also to other reward types, suggesting generalized overactivity of the reward system. The current fMRI study investigated the relationship between general reward sensitivity and eating behavior in normal-weight individuals, while controlling for trait impulsivity. Participants were young adults, some demonstrating ADHD symptoms, allowing for a range of impulsivity profiles. A classical conditioning task was used to measure striatal responses to monetary reward stimuli. Uncontrolled eating scores from the Three Eating Factor Questionnaire were positively correlated with caudate responses to reward predicting cues. This association was not explained by self-reported impulsivity. The current findings provide support for heightened reward anticipation as a neural phenotype contributing to overeating.
\end{abstract}

brain | reward anticipation | reward sensitivity | uncontrolled eating | fMRI caudate | striatum | normal-weight adult | impulsivity |

Correspondence: bado.patricia@gmail.com

Introduction. Attention to factors affecting food intake has grown in recent years as the number of overweight individuals, and rates of obesity, increase worldwide (2015 Obesity Collaborators, 2015). Environmental, genetic and personality factors have all been hypothesized to contribute to overeating and unhealthy weight gain (2015 Obesity Collaborators, 2015; Roberto et al., 2015). Among them is the individuals' tendency for hedonic eating, i.e., food consumption for pleasure beyond energy maintenance (Kenny, 2011). Importantly, hedonic eating plays significant role in food consumption in environments with sufficient food availability (Zheng, Lenard, Shin, I\& Berthoud, 2009).

Individuals differ in sensitivity to food stimuli, e.g., pleasant pictures and smells of food (van der Laan et al., 2011; Fedoroff, Polivy, I\& Herman, 1997). Previous studies have demonstrated that self-reported heightened sensitivity to food reward contributes to palatable food intake and overeating (Appelhans et al., 2011). In neuroimaging studies, individuals diagnosed with Binge Eating Disorder show increased activity to pictures of food in the cortical and subcortical brain regions that have consistently been implicated in reward responses (Rothemund et al., 2007; Schienle, Schäfer, Hermann, I\& Vaitl, 2009). However, it remains unclear whether the heightened sensitivity to reward in these individuals is specific to food or if its generalizes to other types of reward. Individuals with binge eating problems (Bodell et al., 2018) and obese individuals (Balodis et al., 2013) show increased brain activation in response to non-food reward stimuli, such as monetary reward, compared to their non-binge eating or normal weight peers.

In non-disordered samples, individuals who score high on self-report measures of sensitivity to food reward also score high on broader trait reward sensitivity measures (Davis et al., 2007; Davis, Strachan, I\& Berkson, 2004; De Cock et al., 2016). Those reporting high trait reward sensitivity tend to have a higher body weight (Davis et al., 2004; Franken I\& Muris, 2005), report a higher level of food intake and food craving (De Cock et al., 2016; Cepeda-Benito, Gleaves, Williams, I\& Erath, 2000), and show increased brain activation in response to food images (Beaver et al., 2006; Schienle et al., 2009). Together, these findings suggest that eating behavior may be related to non-food specific, general reward sensitivity.

In addition to reward sensitivity, there is evidence indicating that impulsivity contributes to overeating. Trait impulsivity is predictive of heightened food intake in normal weight women (Guerrieri et al, 2007). High rates of binge eating and obesity are reported in attention deficit hyperactivity disorder (ADHD), a disorder often characterized by marked impulsivity (Cortese, Bernardina, I\& Mouren, 2007; Cortese et al., 2016; Davis et al., 2008; Dawe I\& Loxton, 2004; Nazar et al., 2016; Ptacek et al., 2016; Schag, Schönleber, Teufel, Zipfel, I\& Giel, 2013; Schmidt, Körber, de Zwaan, I\& Müller, 2012; Seymour, Reinblatt, Benson, I\& Carnell, 2015). A relationship between impulsivity symptoms and overeating in individuals with ADHD has been documented (Kaisari, Dourish, I\& Higgs, 2017; Nazar et al., 2014). High rates of comorbidity have also been reported between eating disorders and substance use and gambling disorders (Cassin I\& von Ranson, 2007; Dawe I\& Loxton, 2004; García-García et al., 2014; Lesieur I\& Blume, 1993; Wolfe I\& Maisto, 2000). In these disorders of impulse control, altered reward sensitivity has also been documented (Sescousse, Barbalat, Domenech, I\& Dreher, 2013; Tripp I\& Alsop, 2001; Volkow et al., 2010). Such overlap suggests a strong link between reward sensitivity and behavioral impulsivity. This raises a possibility that the association between increased general reward sensitivity 
and overeating may be mediated by impulsivity.

Functional neuroimaging studies have long been used to investigate the neural correlates of reward sensitivity. Activations in the ventral and dorsal striatum have been interpreted as an index of sensitivity to reward and to rewardpredicting cues in both disordered and non-disordered populations (Baroni I\& Castellanos, 2015; Bodell et al., 2018; Dreher, Kohn, Kolachana, Weinberger, I\& Berman, 2009; Furukawa et al., 2014; Knutson I\& Cooper, 2005; Knutson I\& Gibbs, 2007; Knutson I\& Heinz, 2015; O’Doherty, 2004; Wei et al., 2018). The striatum receives significant dopaminergic input, and the neuromodulator dopamine is strongly implicated as the mediator of the brain's reinforcement signal (W. Schultz, Dayan, I\& Montague, 1997; Schultz, 2006). Previous human imaging and experimental non-human animal work together strongly suggest that the level of striatal activation to reward stimuli can be considered a neurobiological index of reward sensitivity.

The current study examined the relationship between nonfood reward sensitivity and eating behavior among young normal-weight adults, with a wide range of impulsivity profiles, in an fMRI task. Sensitivity to monetary reward outcomes and to reward-predicting cues was measured using a classical conditioning paradigm (Furukawa et al., 2018). We hypothesized that increased sensitivity to rewarding stimuli (reward outcomes and/or cues) would be correlated with higher levels of uncontrolled eating, as measured by selfreport (Three Factor Eating Questionnaire (Karlsson, Persson, Sjöström, I\& Sullivan, 2000)). We also examined whether reward sensitivity correlates uniquely with eating behavior, or impulsivity more generally.

Methods. The current study used data collected as part of study on reward sensitivity and ADHD at the D'Or Institute for Research and Education (IDOR) in Rio de Janeiro Brazil, in collaboration with the Okinawa Institute of Science and Technology (OIST) Graduate University. The study (study IRB approval number 377.153) was approved by the IDOR and OIST ethics committees and all participants provided written informed consent before entering the study.

Participants. The study sample included thirty-seven righthanded young adults (21 to 34 years of age, 20 males) recruited at the Federal University of Rio de Janeiro and through investigators' personal contacts (Table 1). All participants belonged to middle and upper socioeconomic classes (Class C1 through A1, http://www.abep.org/). The average BMI of the sample was 23.07, with a range of 18.2 to 29.8. The inclusion criteria for the study were: no current substance abuse, psychotic symptoms, or major depressive or bipolar disorder, or history of any neurological disorder. These were assessed using structured clinical interviews performed by a board-certified psychiatrist (Portuguese version of the Kiddie-Schedule for Affective Disorder and Schizophrenia-PL (KSADS-PL) (Grevet et al., 2005) and SCID (Del-Ben et al., 2001)).

Three of the participants were diagnosed with anxiety disorder, one obsessive-compulsive disorder, seventeen ADHD,
Table 1. Participant demographic information $(n=37)$.

\begin{tabular}{lcc}
\hline & mean & sd \\
\hline Age & 26.46 & 3.41 \\
BMI & 23.07 & 2.71 \\
Education(years) & 16.95 & 1.89 \\
Estimated IQ & 107.43 & 7.37 \\
Socioeconomic (IBGE) & 33.34 & 7.66 \\
Gender & $54 \%$ males & \\
\hline
\end{tabular}

and one individual had a previous diagnosis of binge eating disorder, according to DSM-5 criteria. To check that any observed effects were not driven by individuals with eating disorders, the data were analyzed with and without these two participants. As removing data from these participants did not change the results, their data was retained in the final analyses. Data analyses were carried out for individuals with and without ADHD separately and together. Those who were taking stimulant medication for ADHD symptoms underwent a washout for 2 days prior to the study (see Supplemental Table 1 for participant characteristics of individuals with and without ADHD). The effect of gender was assessed, but there were no significant gender differences on self-report uncontrolled eating behavior. Furthermore, entering gender as a covariate did not change correlational results, so all participants were pooled together for the final analyses.

Self-report behavioral measures. Eating behavior was assessed using the 21 item Three-Factor Eating Questionnaire (TFEQ, Cappelleri et al., 2009; Karlsson et al., 2000). The Portuguese version of this measure demonstrates adequate reliability and validity (Natacci I\& Ferreira Júnior, 2011). The questionnaire's three factors are: uncontrolled eating $(9$ items), emotional eating (6 items) and cognitive restraint (6 items). Participants respond on a four-point Likert scale for items 1-20, and on an eight-point rating scale for item 21 (cognitive restraint item). The uncontrolled eating factor was of particular interest, in this study, because of its close relationship to hedonic eating and impulsivity (Vainik, Neseliler, Konstabel, Fellows, I\& Dagher, 2015; Yeomans, Leitch, I\& Mobini, 2008). The factor includes items such as "Sometimes when I start eating, I just can't seem to stop."; "When I see something that looks very delicious, I often get so hungry that I have to eat right away." "I'm always hungry enough to eat at any time".

Trait impulsivity was assessed using the Impulsive Behavior Scale UPPS (Whiteside I\& Lynam, 2001). The scale assumes a heterogeneous construct of impulsivity and comprises four dimensions: urgency, sensation seeking, (lack of) premeditation and (lack of) perseverance. The Portuguese version of the measure demonstrates adequate reliability and validity (Sediyama et al., 2017). Previous studies have demonstrated correlations of the UPPS dimensions with reward sensitivity (Carlson, Pritchard, I\& Dominelli, 2013) and eating behavior (Moreno-López, Soriano-Mas, Delgado-Rico, Rio-Valle, I\& Verdejo-García, 2012). 


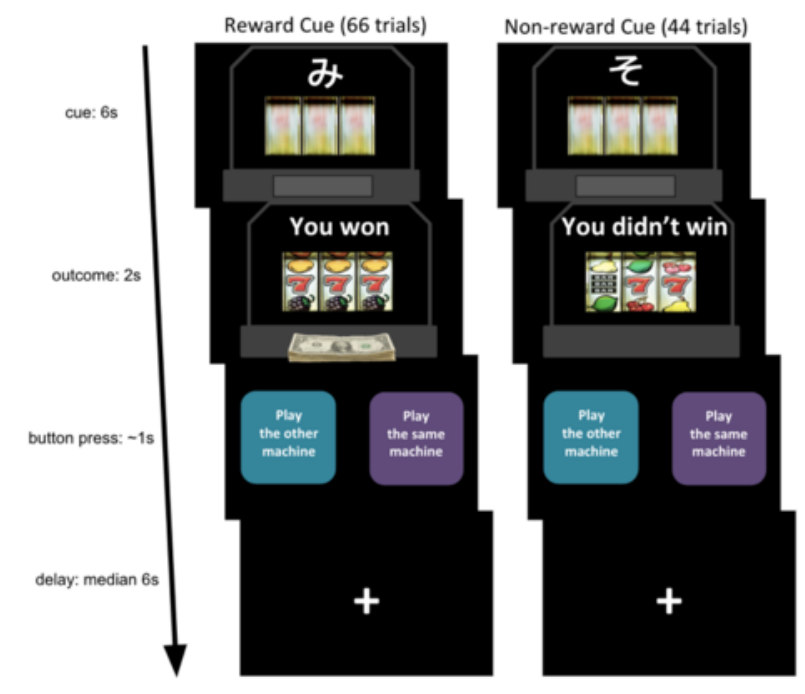

Fig. 1. Classical conditioning fMRI paradigm using monetary reward. One of two previously neutral stimuli (Reward Cue or Non-reward Cue) was followed by an outcome stimulus (reward or non-reward) after a 6-second delay. The reward cue was followed by the delivery of the reward 66.7 percent of the time. The non-reward cue was always followed by non-reward. Participants were asked to suggest to the computer which machine to play next, the actual presentation was random. This "choice" was included to maintain attention to the task. The length of the inter-trial delay was varied using Poisson distribution (median 6s).

fMRI Experimental Paradigm. A classical conditioning fMRI task was used to evaluate blood-oxygen-leveldependent (BOLD) responses to a reward-predicting cue and reward outcome (Figure 1, Furukawa et al. 2018). Pictures of two slot machines were displayed one at a time, each with a single cue (Cue A or Cue B). The machines spun for 6 seconds and stopped at a win or non-win position (reward or non-reward) for 2 seconds. The stimuli presentation was event-related, in a semi-random order. Cue A was followed by reward $2 / 3$ of the time ( 44 trials), by non-reward $1 / 3$ of the time (22 trials), and Cue B was never followed by reward (44 trials). Participants completed a brief trial run before entering the MRI scanner.

Data analysis. Questionnaire and fMRI data were used to examine the 1) relationship between self-report eating behavior and impulsivity, 2) relationship between self-report eating behavior and BOLD responses to non-food reward stimuli (reward sensitivity), and 3) contribution of self-report impulsivity to the relationship between eating behavior and reward sensitivity. Pearson correlations were used to examine associations between behavior scales, using SPSS (https://www.ibm.com/analytics/spss-statistics-software).

The functional images were analyzed using Statistical Parametric Mapping software (SPM12; http://www.fil.ion.ucl.ac.uk/spm/software/spm12/). Preprocessing was completed using realignment, slice-time correction, co-registration, and normalization to the standard MNI template resulting in the reconstructed functional images with voxel dimensions of $3 \mathrm{~mm}$. Images were spatially smoothed (6 mm full-width half-maximum Gaussian spatial kernel). Boxcar functions at stimulus onset for specified events were convolved with the hemodynamic response function with autocorrelation correction $(\mathrm{AR}(1))$ and high-pass
Table 2. Scores on self-report questionnaires of eating behavior (TFEQ) and impulsivity (UPPS).

\begin{tabular}{lccc}
\hline & mean & sd & range \\
\hline TFEQ & & & \\
Uncontrolled Eating & 17.88 & 5.15 & $11-34$ \\
Emotional Eating & 10.90 & 4.01 & $6-20$ \\
Cognitive Restraint & 13.50 & 4.76 & $6-22$ \\
UPPS & & & \\
Urgency & 27.31 & 7.55 & $15-44.5$ \\
Sensation Seeking & 31.86 & 8.33 & $16-48$ \\
Premeditation & 20.38 & 4.43 & $12-29.7$ \\
Perseverance & 21.78 & 5.89 & $13-34$ \\
\hline
\end{tabular}

filtering (128s) for each participant. Condition-specific firstlevel regressors (Cue A, Cue B, Reward and Non-reward) were entered with six movement parameters.

Participants' ratings on the TFEQ factors were entered as covariates, one at a time, in the second-level GLM analysis to examine the relationship between BOLD responses to reward cues and reward outcome and each TFEQ factor. All analyses were performed using whole-brain, $\mathrm{p}<.001$, uncorrected, minimum cluster size $(\mathrm{k}=10)$. An $10 \mathrm{~mm}$ sphere for caudate (MNI: 8202 (Liu, Hairston, Schrier, I\& Fan, 2011)) was used for small volume correction and for parameter estimate plots. Parameter estimates from the ROI (caudate) sphere was extracted using rfxplot toolbox for SPM (http://rfxplot.sourceforge.net). Correlations between the parameter estimates and UPPS scales were examined in SPSS. Further, an ANOVA and partial correlation using SPSS GLM examined the relationship between the ROI parameter estimates and the uncontrolled eating scale while controlling for UPPS dimensions.

\section{Results.}

Relationship between eating behavior and impulsivity. Correlations between the TEFQ and UPPS scales were examined (Supplemental Table 2). Uncontrolled eating scores were significantly positively correlated with Urgency $(r=.36$, $\mathrm{p}<.03)$ and lack of Perseverance $(\mathrm{r}=.34, \mathrm{p}<.04)$, i.e., higher uncontrolled eating scores were associated with increased urgency and increased lack of perseverance. TFEQ and UPPS scores are presented in Table 2 .

\section{Relationship between eating behavior and BOLD re-} sponses to reward stimuli. Higher uncontrolled eating scores were associated with increased caudate responses to the reward-predicting cue across participants $(\mathrm{z}=3.85$, pFWE $<.01$, thresholded at whole-brain uncorrected $\mathrm{p}<$ $.001, \mathrm{k}=10$, Figure $2 \mathrm{a}$ ). The caudate and temporoparietal junction responses (Table 3 ) were considerably selective, since only two activation clusters were observed across the whole brain. No surviving suprathreshold cluster was observed in response to the reward-predicting cue when association with the other two TFEQ dimensions (emotional eating and cognitive restraint) were entered as covariates. No surviving suprathreshold cluster was observed in response to 


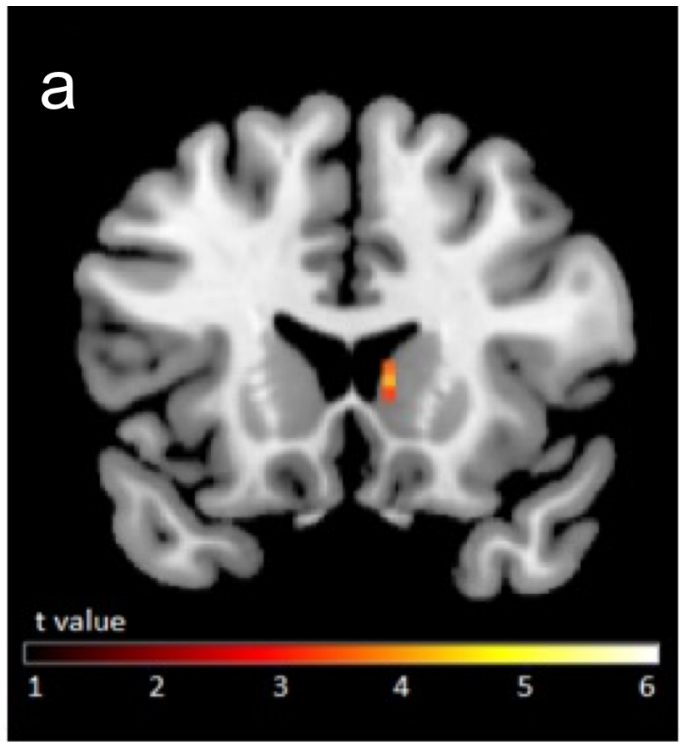

\section{b}

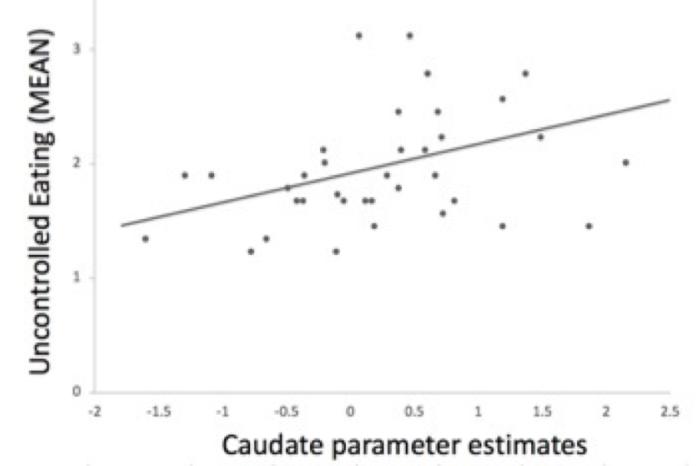

Fig. 2. Correlations between caudate responses during reward anticipation and Uncontrolled Eating scores for all participants. a) Brain second-level map for the reward-predicting cue condition with participants' Uncontrolled Eating scores entered as covariate, gray matter mask applied for visualization. b) Correlation between beta values from caudate local maxima and participants' Uncontrolled Eating scores; the graph in (b) is provided for illustrative purposes only and was not used for statistical inferences.

reward outcome, in relation to any of the TFEQ dimensions at whole brain $\mathrm{p}<0.001, \mathrm{k}=10$.

Relationship between uncontrolled eating and caudate responses to reward cues, controlling for impulsivity. No significant correlation was found between caudate parameter estimates and UPPS dimensions. A regression analysis was performed with the caudate parameter estimates as the dependent variable and the uncontrolled eating and two UPPS scores (Urgency and Perseverance, which were correlated with uncontrolled eating) as predictor variables, entered simultaneously. The model accounted for significant variance in the caudate responses $(\mathrm{F}(3,36)=2.98, \mathrm{R} 2=.21, \mathrm{p}<.05)$. The partial correlation between the caudate response and uncontrolled eating was significant $(\mathrm{r}=.40, \mathrm{p}<.05)$ while controlling for impulsivity (UPPS scores). Partial correlations for Urgency $(r=.15)$ and lack of Perseverance $(r=-.28)$ were not significant.
Table 3. Brain regions demonstrating significant BOLD responses to reward cue associated with Uncontrolled Eating scores, entered as a second level covariate. Clusters are shown at an uncorrected threshold p.001. Small volume corrections using $10 \mathrm{~mm}$ sphere from independent meta-analysis coordinates were applied. No suprathreshold cluster were observed for reward delivery contrast and TFEQ scores.

\begin{tabular}{lcccc}
\hline Region & $\mathrm{k}$ & $\mathrm{Z}$ value & P FWE & MNI \\
\hline Caudate & 18 & 3.85 & $<0.01$ & $6,20,8$ \\
TPJ & 13 & 4.06 & NA & $-48,-49,23$ \\
\hline
\end{tabular}

Contribution of ADHD to relationship between uncontrolled eating, caudate responses, and impulsivity. The inclusion of participants with ADHD allowed for a wide range of impulsivity profiles in the current sample. The effects of ADHD on the relationship between uncontrolled eating, caudate responses and impulsivity were explored, given the documented high comorbidity rates between ADHD and Eating Disorder (Cortese et al., 2016). There was no significant difference between individuals with and without ADHD on the Uncontrolled eating scale, or other TEFQ scales. The UPPS (lack of) Perseverance score was significantly higher for those with ADHD (t $(35)=6.39,<.001)$, while the correlation between Uncontrolled eating and Urgency was stronger for individuals with ADHD $(r=.68)$ than those without ADHD $(r=-.10)$ (Fisher $r-t o-z=2.62, p<.01$ ) (Supplemental Table 3).

There was no significant difference in the mean beta values from the caudate local maxima in participants with and without ADHD. The correlation between the caudate beta values and uncontrolled eating scores was stronger for participants with ADHD $(r=.60)$ than those without ADHD $(r=$ .27) (Supplemental Figure 1); however, the magnitudes of the correlations were not significantly different (Fisher r-to- $z=$ 1.14). In the regression analysis, when the ADHD diagnosis was entered in the first step (i.e., controlling for ADHD), the partial correlation between uncontrolled eating and caudate responses remained significant $(\mathrm{r}=.40, \mathrm{p}<.05)$.

Discussion. The current study examined the relationship between self-reported eating behavior and sensitivity to nonfood reward stimuli in a young normal weight adult sample with a wide range of impulsivity profiles. Caudate responses to previously neutral, reward-predicting cues were associated with higher self-reported uncontrolled eating behavior. No significant relationship was observed between responses to reward outcomes and self-reported eating behavior. To our knowledge, this study is the first to examine BOLD responses to monetary rewards and conditioned cues in the context of eating behavior in normal-weight adults. Such relationships have previously been demonstrated in obese or eating disordered samples (Balodis et al., 2013; Bodell et al., 2018).

The association between monetary reward cue sensitivity and uncontrolled eating is consistent with previous literature indicating that trait reward sensitivity, as measured by selfreport, is implicated in overeating (Davis et al., 2007, 2004; De Cock et al., 2016) and palatable food intake (Appelhans et al., 2011). Our findings with normal-weight participants, 
demonstrating heightened brain activation in response to nonfood reward cues, are also consistent with previous studies reporting increased striatal responses during the anticipatory phase of the Monetary Incentive Delay task in adolescents with binge eating episodes (Bodell et al., 2018) and obese adults (Balodis et al., 2013).

The association between the caudate responses to reward cues and uncontrolled eating was not explained by levels of impulsivity. Although the urgency and lack of perseverance subscale scores correlated with uncontrolled eating, these traits did not contribute to caudate responses nor changed the degree of association between the caudate responses and uncontrolled eating. These results persisted despite the inclusion of adults with ADHD symptoms in the sample, some demonstrating increased behavioral impulsivity. Taken together, these data indicate altered reward sensitivity makes a unique contribution to overeating, which is not explained by self-reported impulsivity. The current study found an association between uncontrolled eating and BOLD responses in the dorsomedial striatum only, more specifically in the head of the left caudate nucleus. Increased activation in the same dorsal caudate cluster as the current study $(6 \mathrm{~mm}$ sphere from our MNI local maxima, http://neurosynth.org/) has been reported in response to food images in obese and overweight individuals (Filbey, Myers, I\& Dewitt, 2012; Nummenmaa et al., 2012; Rothemund et al., 2007), and to monetary rewards in adolescents demonstrating binge eating (Bodell et al., 2018).

The absence of an association between ventral striatum activation and self-reported uncontrolled eating was surprising. The ventral striatum is densely populated with dopaminergic neurons, and increased activation in the ventral striatum has previously been reported in response to food images in those reporting high reward sensitivity (Beaver et al., 2006), and to monetary rewards in obese individuals (Balodis et al., 2013). We had therefore expected increased ventral striatal responses to reward stimuli in the current study. Increased activation in the left temporoparietal junction was also associated with uncontrolled eating in the current study. This region has been implicated in sensitivity to cigarette cues in smokers (Xu, Aron, Westmaas, Wang, I\& Sweet, 2014, http://neurosynth.org/). However, the temporoparietal junction was not among the current study's a priori regions for analysis, therefore we limited the interpretation of this activation.

Exploratory analysis examined the effects of ADHD. As expected, participants with the disorder demonstrated higher trait impulsivity. The relationship between the caudate responses to reward cues and uncontrolled eating were stronger among individuals with ADHD, compared to those without the disorder. However, the difference in the magnitudes of the correlations did not reach statistical significance. Controlling for the diagnosis of ADHD did not change the percentage of variance uniquely shared by the caudate responses and uncontrolled eating. This finding further strengthens our confidence that the link between reward sensitivity and eating behavior is not dependent on trait impulsivity.
Our results indicate that the relationship between a heightened sensitivity to non-food reward cues and uncontrolled eating extends to normal-weight adults, rather than being specific to those with an eating disorder or are overweight. In individuals who are more sensitive to rewarding stimuli in general, enjoyable experiences, including eating, may be enhanced. For these individuals, eating behavior may be more strongly reinforced by pleasurable eating experiences and cues that remind them of the experiences. Subsequent willingness to eat may thus be motivated by an enhanced anticipation of reward, rather than by need for energy maintenance.

Conclusion and perspectives. The current results, together with previous literature, suggest that increased sensitivity to reward anticipation is an important mechanism underlying overeating, irrespective of weight, eating disorder symptoms or levels of impulsivity. The relationship between heightened striatal responses to reward-predicting cues and greater likelihood of uncontrolled eating found in the current study point to a possible neurobiological vulnerability in individuals who report eating beyond satiety. In everyday life, cues signaling the availability of food are consistently present. These environmental cues affect the behavior of all individuals; however, the degree to which such reward cues exert control over behavior and encourage eating may vary. Improving understanding of how reward sensitivity affects eating behavior is important, especially in today's society where many individuals have easy access to inexpensive, palatable food. Rapidly raising rates of obesity, and the population average weight, argue for greater attention to the effects of environmental cues on eating behavior.

\section{Bibliography}

2015 Obesity Collaborators, G. B. D. (2015). Health effects of overweight and obesity in 195 countries over 25 years. New England Journal of. Retrieved from https://www.nejm.org/doi/full/10.1056/NEJMoa1614362 Appelhans, B. M., Woolf, K., Pagoto, S. L., Schneider, K. L., Whited, M. C., I\& Liebman, R. (2011). Inhibiting food reward: delay discounting, food reward sensitivity, and palatable food intake in overweight and obese women. Obesity, 19(11), 2175-2182.

Balodis, I. M., Kober, H., Worhunsky, P. D., White, M. A., Stevens, M. C., Pearlson, G. D., ... Potenza, M. N. (2013). Monetary reward processing in obese individuals with and without binge eating disorder. Biological Psychiatry, 73(9), 877-886.

Baroni, A., I\& Castellanos, F. X. (2015). Neuroanatomic and cognitive abnormalities in attention-deficit/hyperactivity disorder in the era of "high definition" neuroimaging. Current Opinion in Neurobiology, 30, 1-8.

Beaver, J. D., Lawrence, A. D., van Ditzhuijzen, J., Davis, M. H., Woods, A., I\& Calder, A. J. (2006). Individual differences in reward drive predict neural responses to images of food. The Journal of Neuroscience: The Official Journal of the Society for Neuroscience, 26(19), 5160-5166. 
Bodell, L. P., Wildes, J. E., Goldschmidt, A. B., Lepage, R., Keenan, K. E., Guyer, A. E., ... Forbes, E. E. (2018). Associations Between Neural Reward Processing and Binge Eating Among Adolescent Girls. The Journal of Adolescent Health: Official Publication of the Society for Adolescent Medicine, 62(1), 107-113.

Cappelleri, J. C., Bushmakin, A. G., Gerber, R. A., Leidy, N. K., Sexton, C. C., Lowe, M. R., I\& Karlsson, J. (2009). Psychometric analysis of the Three-Factor Eating QuestionnaireR21: results from a large diverse sample of obese and nonobese participants. International Journal of Obesity, 33(6), 611-620.

Carlson, S. R., Pritchard, A. A., I\& Dominelli, R. M. (2013). Externalizing behavior, the UPPS-P Impulsive Behavior scale and Reward and Punishment Sensitivity. Personality and Individual Differences, 54(2), 202-207.

Cassin, S. E., I\& von Ranson, K. M. (2007). Is binge eating experienced as an addiction? Appetite, 49(3), 687-690.

Cepeda-Benito, A., Gleaves, D. H., Williams, T. L., I\& Erath, S. A. (2000). The development and validation of the state and trait food-cravings questionnaires. Behavior Therapy, 31(1), 151-173.

Cortese, S., Bernardina, B. D., I\& Mouren, M.-C. (2007). Attention-deficit/hyperactivity disorder (ADHD) and binge eating. Nutrition Reviews, 65(9), 404-411.

Cortese, S., Moreira-Maia, C. R., St Fleur, D., MorcilloPeñalver, C., Rohde, L. A., I\& Faraone, S. V. (2016). Association Between ADHD and Obesity: A Systematic Review and Meta-Analysis. The American Journal of Psychiatry, 173(1), 34-43.

Davis, C., Levitan, R. D., Carter, J., Kaplan, A. S., Reid, C., Curtis, C., ... Kennedy, J. L. (2008). Personality and eating behaviors: a case-control study of binge eating disorder. The International Journal of Eating Disorders, 41(3), 243-250.

Davis, C., Patte, K., Levitan, R., Reid, C., Tweed, S., I\& Curtis, C. (2007). From motivation to behaviour: a model of reward sensitivity, overeating, and food preferences in the risk profile for obesity. Appetite, 48(1), 12-19.

Davis, C., Strachan, S., I\& Berkson, M. (2004). Sensitivity to reward: implications for overeating and overweight. Appetite, 42(2), 131-138. Dawe, S., I\& Loxton, N. J. (2004). The role of impulsivity in the development of substance use and eating disorders. Neuroscience and Biobehavioral Reviews, 28(3), 343-351.

De Cock, N., Van Lippevelde, W., Vervoort, L., Vangeel, J., Maes, L., Eggermont, S., ... Van Camp, J. (2016). Sensitivity to reward is associated with snack and sugar-sweetened beverage consumption in adolescents. European Journal of Nutrition, 55(4), 1623-1632.

Del-Ben, C. M., Vilela, J. A. A., Crippa, J. A. de S., Hallak, J. E. C., Labate, C. M., I\& Zuardi, A. W. (2001). Confiabilidade da "Entrevista Clínica Estruturada para o DSM-IV - Versão Clínica" traduzida para o português. Revista Brasileira de Psiquiatria, 23(3), 156-159.

Dreher, J. C., Kohn, P., Kolachana, B., Weinberger, D. R., I\& Berman, K. F. (2009). Variation in dopamine genes influences responsivity of the human reward system. Proceedings of the National Academy of Sciences, 106(2), 617-622.

Fedoroff, I. C., Polivy, J., I\& Herman, C. P. (1997). The effect of pre-exposure to food cues on the eating behavior of restrained and unrestrained eaters. Appetite, 28(1), 33-47.

Filbey, F. M., Myers, U. S., I\& Dewitt, S. (2012). Reward circuit function in high BMI individuals with compulsive overeating: similarities with addiction. NeuroImage, 63(4), 1800-1806.

Franken, I. H. A., I\& Muris, P. (2005). Individual differences in reward sensitivity are related to food craving and relative body weight in healthy women. Appetite, 45(2), 198-201.

Furukawa, E., Bado, P., Tripp, G., Mattos, P., Wickens, J. R., Bramati, I. E., ... Moll, J. (2014). Abnormal striatal BOLD responses to reward anticipation and reward delivery in ADHD. PloS One, 9(2), e89129.

Furukawa E, Costa R, Bado P, Hoefle S, Vigne P, Monteiro M, Tripp G, Wickens J, Moll J, I\& Mattos P. (September, 2018). Methylphenidate effects on correlated fMRI activity during reward processing in adults with ADHD. 5th EUNETHYDIS International Conference, Edinburgh, Scotland. García-García, I., Horstmann, A., Jurado, M. A., Garolera, M., Chaudhry, S. J., Margulies, D. S., Neumann, J. (2014). Reward processing in obesity, substance addiction and nonsubstance addiction. Obesity Reviews: An Official Journal of the International Association for the Study of Obesity, 15(11), 853-869.

Grevet, E. H., Bau, C. H. D., Salgado, C. A. I., Ficher, A., Victor, M. M., Garcia, C., ... Belmonte-de-Abreu, P. (2005). Interrater reliability for diagnosis in adults of attention deficit hyperactivity disorder and oppositional defiant disorder using K-SADS-E. Arquivos de Neuro-Psiquiatria, 63(2A), 307-310.

Guerrieri, R., Nederkoorn, C., Stankiewicz, K., Alberts, H., Geschwind, N., Martijn, C., I\& Jansen, A. (2007). The influence of trait and induced state impulsivity on food intake in normal-weight healthy women. Appetite, 49(1), 66-73.

Kaisari, P., Dourish, C. T., I\& Higgs, S. (2017). Attention Deficit Hyperactivity Disorder (ADHD) and disordered eating behaviour: A systematic review and a framework for future research. Clinical Psychology Review, 53, 109-121.

Karlsson, J., Persson, L. O., Sjöström, L., I\& Sullivan, M. (2000). Psychometric properties and factor structure of the Three-Factor Eating Questionnaire (TFEQ) in obese men and women. Results from the Swedish Obese Subjects (SOS) study. International Journal of Obesity and Related Metabolic Disorders: Journal of the International Association for the Study of Obesity, 24(12), 1715-1725.

Kenny, P. J. (2011). Reward mechanisms in obesity: new insights and future directions. Neuron, 69(4), 664-679.

Knutson, B., I\& Cooper, J. C. (2005). Functional magnetic resonance imaging of reward prediction. Current Opinion in Neurology, 18(4), 411.

Knutson, B., I\& Gibbs, S. E. B. (2007). Linking nucleus accumbens dopamine and blood oxygenation. Psychopharmacology, 191(3), 813-822.

Knutson, B., I\& Heinz, A. (2015). Probing psychiatric symptoms with the monetary incentive delay task. Biological Psy- 
chiatry, 77(5), 418-420.

Lesieur, H. R., I\& Blume, S. B. (1993). Pathological gambling, eating disorders, and the psychoactive substance use disorders. Journal of Addictive Diseases, 12(3), 89-102.

Liu, X., Hairston, J., Schrier, M., I\& Fan, J. (2011). Common and distinct networks underlying reward valence and processing stages: a meta-analysis of functional neuroimaging studies. Neuroscience and Biobehavioral Reviews, 35(5), 1219-1236.

Moreno-López, L., Soriano-Mas, C., Delgado-Rico, E., RioValle, J. S., I\& Verdejo-García, A. (2012). Brain structural correlates of reward sensitivity and impulsivity in adolescents with normal and excess weight. PloS One, 7(11), e49185.

Natacci, L. C., I\& Ferreira Júnior, M. (2011). The three factor eating questionnaire - R21: translation and administration to Brazilian women. Revista de Nutrição, 24(3), 383-394.

Nazar, B. P., Bernardes, C., Peachey, G., Sergeant, J., Mattos, P., I\& Treasure, J. (2016). The risk of eating disorders comorbid with attention-deficit/hyperactivity disorder: A systematic review and meta-analysis. The International Journal of Eating Disorders, 49(12), 1045-1057.

Nazar, B. P., Suwwan, R., de Sousa Pinna, C. M., Duchesne, M., Freitas, S. R., Sergeant, J., I\& Mattos, P. (2014). Influence of attention-deficit/hyperactivity disorder on binge eating behaviors and psychiatric comorbidity profile of obese women. Comprehensive Psychiatry, 55(3), 572-578.

Nummenmaa, L., Hirvonen, J., Hannukainen, J. C., Immonen, H., Lindroos, M. M., Salminen, P., I\& Nuutila, P. (2012). Dorsal striatum and its limbic connectivity mediate abnormal anticipatory reward processing in obesity. PloS One, 7(2), e31089.

O'Doherty, J. P. (2004). Reward representations and reward-related learning in the human brain: insights from neuroimaging. Current Opinion in Neurobiology, 14(6), 769-776.

Ptacek, R., Stefano, G. B., Weissenberger, S., Akotia, D., Raboch, J., Papezova, H., ... Goetz, M. (2016). Attention deficit hyperactivity disorder and disordered eating behaviors: links, risks, and challenges faced. Neuropsychiatric Disease and Treatment, 12, 571-579.

Roberto, C. A., Swinburn, B., Hawkes, C., Huang, T. T.-K., Costa, S. A., Ashe, M., ... Brownell, K. D. (2015). Patchy progress on obesity prevention: emerging examples, entrenched barriers, and new thinking. The Lancet, 385(9985), 2400-2409.

Rothemund, Y., Preuschhof, C., Bohner, G., Bauknecht, H.C., Klingebiel, R., Flor, H., I\& Klapp, B. F. (2007). Differential activation of the dorsal striatum by high-calorie visual food stimuli in obese individuals. NeuroImage, 37(2), 410-421.

Schag, K., Schönleber, J., Teufel, M., Zipfel, S., I\& Giel, K. E. (2013). Food-related impulsivity in obesity and binge eating disorder-a systematic review. Obesity Reviews: An Official Journal of the International Association for the Study of Obesity, 14(6), 477-495.

Schienle, A., Schäfer, A., Hermann, A., I\& Vaitl, D. (2009). Binge-eating disorder: reward sensitivity and brain activation to images of food. Biological Psychiatry, 65(8), 654-661. Schmidt, F., Körber, S., de Zwaan, M., I\& Müller, A. (2012). Impulse control disorders in obese patients. European Eating Disorders Review: The Journal of the Eating Disorders Association, 20(3), e144-e147. Schultz, W. (2006). Behavioral theories and the neurophysiology of reward. Annual Review of Psychology, 57, 87-115.

Schultz, W., Dayan, P., I\& Montague, P. R. (1997). A neural substrate of prediction and reward. Science, 275(5306), 1593-1599.

Sediyama, C. Y. N., Moura, R., Garcia, M. S., da Silva, A. G., Soraggi, C., Neves, F. S., ... Malloy-Diniz, L. F. (2017). Factor Analysis of the Brazilian Version of UPPS Impulsive Behavior Scale. Frontiers in Psychology, 8, 622.

Sescousse, G., Barbalat, G., Domenech, P., I\& Dreher, J.-C. (2013). Imbalance in the sensitivity to different types of rewards in pathological gambling. Brain: A Journal of Neurology, 136(Pt 8), 2527-2538.

Seymour, K. E., Reinblatt, S. P., Benson, L., I\& Carnell, S. (2015). Overlapping neurobehavioral circuits in ADHD, obesity, and binge eating: evidence from neuroimaging research. CNS Spectrums, 20(4), 401-411.

Torrubia, R., Ávila, C., Moltó, J., I\& Caseras, X. (2001). The Sensitivity to Punishment and Sensitivity to Reward Questionnaire (SPSRQ) as a measure of Gray's anxiety and impulsivity dimensions. Personality and Individual Differences, 31(6), 837-862.

Tripp, G., I\& Alsop, B. (2001). Sensitivity to reward delay in children with attention deficit hyperactivity disorder (ADHD). Journal of Child Psychology and Psychiatry, and Allied Disciplines, 42(5), 691-698.

Vainik, U., Neseliler, S., Konstabel, K., Fellows, L. K., I\& Dagher, A. (2015). Eating traits questionnaires as a continuum of a single concept. Uncontrolled eating. Appetite, 90, 229-239.

van der Laan, L. N., de Ridder, D. T. D., Viergever, M. A., I\& Smeets, P. A. M. (2011). The first taste is always with the eyes: a meta-analysis on the neural correlates of processing visual food cues. NeuroImage, 55(1), 296-303.

Volkow, N. D., Wang, G.-J., Fowler, J. S., Tomasi, D., Telang, F., I\& Baler, R. (2010). Addiction: decreased reward sensitivity and increased expectation sensitivity conspire to overwhelm the brain's control circuit. BioEssays: News and Reviews in Molecular, Cellular and Developmental Biology, 32(9), 748-755.

Wei, S., Zheng, Y., Li, Q., Dai, W., Sun, J., Wu, H., I\& Liu, X. (2018). Enhanced neural responses to monetary rewards in methamphetamine use disordered individuals compared to healthy controls. Physiology I\& Behavior. https://doi.org/10.1016/j.physbeh.2018.08.003

Whiteside, S. P., I\& Lynam, D. R. (2001). The Five Factor Model and impulsivity: using a structural model of personality to understand impulsivity. Personality and Individual Differences, 30(4), 669-689.

Wolfe, W. L., I\& Maisto, S. A. (2000). The relationship between eating disorders and substance use: moving beyond co-prevalence research. Clinical Psychology Review, 20(5), 
$617-631$.

Xu, X., Aron, A., Westmaas, J. L., Wang, J., I\& Sweet, L. H. (2014). An fMRI study of nicotine-deprived smokers' reactivity to smoking cues during novel/exciting activity. PloS One, 9(4), e94598.

Yeomans, M. R., Leitch, M., I\& Mobini, S. (2008). Impulsivity is associated with the disinhibition but not restraint factor from the Three Factor Eating Questionnaire. Appetite, 50(23), 469-476.

Zheng, H., Lenard, N. R., Shin, A. C., I\& Berthoud, H.-R. (2009). Appetite control and energy balance regulation in the modern world: reward-driven brain overrides repletion signals. International Journal of Obesity, 33 Suppl 2, S8-S13.

Zink, Caroline F., Giuseppe Pagnoni, Megan E. MartinSkurski, Jonathan C. Chappelow, and Gregory S. Berns. 2004. "Human Striatal Responses to Monetary Reward Depend on Saliency." Neuron 42 (3): 509-17. 